\title{
La musique dans le rite et la musique comme rite dans le candomblé brésilien
}

\section{Tiago de Oliveira Pinto}

Traducteur : Isabelle Schulte-Tenckhoff

\section{OpenEdition \\ Journals}

\section{Édition électronique}

URL : http://journals.openedition.org/ethnomusicologie/2428

ISSN : 2235-7688

Éditeur

ADEM - Ateliers d'ethnomusicologie

Édition imprimée

Date de publication : 1 janvier 1992

Pagination : 53-70

ISBN : 978-2-8257-0456-1

ISSN : 1662-372X

Référence électronique

Tiago de Oliveira Pinto, « La musique dans le rite et la musique comme rite dans le candomblé

brésilien », Cahiers d'ethnomusicologie [En ligne], 5| 1992, mis en ligne le 15 décembre 2011, consulté le 10 décembre 2020. URL : http://journals.openedition.org/ethnomusicologie/2428 


\title{
LA MUSIQUE DANS LE RITE \\ ET LA MUSIQUE COMME RITE \\ DANS LE CANDOMBLÉ BRÉSILIEN*
}

\author{
Tiago de Oliveira Pinto
}

Dans la plupart des systèmes religieux, la musique occupe un rôle important, sinon central pour la «mise en condition sensorielle» de principes spirituels et mythiques. Mais jusqu'à quel point peut-on considérer la musique comme un élément autonome - et de ce fait susceptible d'être abstrait - du rite religieux? La musique prise au sens le plus général, ne constitue-t-elle pas plutôt une dramatisation de l'acte religieux?

Dans le candomblé, un culte afro-brésilien pratiqué à Bahia mais aussi ailleurs au Brésil, la musique et la danse sont des moyens efficaces de représentation du sacré en vue de la transmission de contenus religieux; elles sont indispensables à l'établissement de liens entre le monde profane et la sphère spirituelle des orixás (ou santos). Après quelques réflexions générales, on tentera d'illustrer, à l'aide d'un exemple de cérémonie, la question de la musique dans le rite et en tant que rite.

\section{MUSIQUE RITUELLE}

Dans la terminologie indigène relative au candomblé, le rôle central de la musique, y compris son rapport intime à la danse, est toujours mis en évidence, bien que les termes portugais de música et dança n'y figurent presque jamais. On désigne généralement la cérémonie publique ou le rite dans le candomblé par le terme de toque, appellation «musicale» car toque se réfère au jeu du tambour, et son pluriel toques, aux formules rythmiques spécifiques. La traduction exacte de tocar candomblé est donc «jouer le candomblé», mais elle comporte également l'idée de la pratique publique d'un rite religieux.

\section{Fonctions et contenus symboliques}

La musique religieuse est jouée à diverses occasions et remplit plusieurs fonctions. Dans le candomblé, les légendes mythiques (lendas) se rapportant au pan-

Traduit de l'allemand par Isabelle Schulte-Tenckhoff. 
théon des orixás sont symbolisées par la musique; mais elle crée aussi un lien direct avec l'univers spirituel. Dans le rite religieux, la musique est chargée de symboles. Par ce qu'elle représente, elle ouvre la voie à une "réalité sacrée» sinon inaccessible à la plupart des gens. Ce sont les normes du système religieux et les conventions léguées par la tradition au sein de la communauté, qui confèrent à la musique son contenu fonctionnel et symbolique. Mais en même temps, ce contenu prend une part directe à ce qu'il symbolise: la mauvaise utilisation d'un chant sacré ou d'une formule rythmique au tambour porterait atteinte à la sphère spirituelle qu'évoque le répertoire du candomblé.

Dans le candomblé, la pratique musicale va donc plus loin que la simple glorification du divin telle qu'on la connaît de la messe catholique. Cette affirmation s'applique non seulement à la forme la plus répandue du candomblé traditionnel (appelée candomblé ketu), avec son panthéon d'orixás qui s'inspire fortement d'éléments ouest-africains d'origine yoruba et son langage liturgique (y compris les paroles des chants), mais encore au candomblé dit angola, dont la terminologie liturgique indique une provenance d'Afrique centrale, notamment de cultures bantoues ${ }^{1}$.

\section{Cantigas et toques}

Quand ils parlent de la musique, les adeptes du candomblé ne manquent pas d'évoquer le couple cantigas/toques, soit chants et rythmes. Les textes des premières sont d'une importance cruciale, car ils expliquent, pour ainsi dire, le déroulement du rite. Quant au langage des tambours (fala dos atabaques), on croit qu'il atteint le monde surnaturel, qu'il appelle et interpelle les orixás que les médiums initiés personnifient dans la seconde partie de la cérémonie publique. La connaissance approfondie des chants et des formules rythmiques est généralement la prérogative des personnalités haut placées du candomblé, dont notamment les musiciens (alabês). Ce savoir musical donne un accès important au candomblé et assure le respect des autres fidèles.

On distingue deux catégories fondamentales de cantigas, dites de fundamento (de roncó, etc.) et de barracão. Les premières, qu'on chante dans les roncós, c'est-à-dire les pièces secrètes de la maison du candomblé, inaccessibles aux gens de l'extérieur, comprennent le répertoire des rites d'initiation (feitura) ou sacrificiels (bori). Les secondes tirent leur nom de la salle principale (barracão); elles couvrent le reste du répertoire, en particulier les diverses invocations d'orixás.

Au sujet des différents nações du candomblé, voir par exemple Bastide (1961), Costa Lima (1976) et Dantas (1988). 


\section{CANTIGAS DE CANDOMBLÉ}

cantigas de barracão

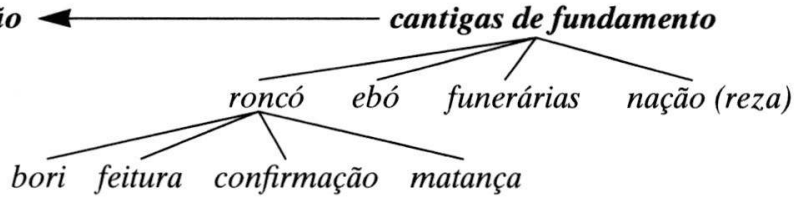

xirê 1ère partie de la cérémonie publique; invocation des santos

dar rum $\quad 2^{\mathrm{e}}$ partie de la cérémonie publique; la sphère spirituelle s'est élargie dans la salle principale

roncó pièce sacrée dans la maison du candomblé

ebó offrande

funeral rite funéraire

nação «nation», groupement au sein de la religion du candomblé

bori offrande de sang pour la tête

feitura initiation

confirmação «confirmation", forme particulière d'initiation

matança sacrifice d'un animal

\section{La musique comme élément d'ordre}

La musique ordonne et structure tout le déroulement du rite. Pendant la réclusion des novices (abiã) dans le roncó, la progression cyclique des moments rituels est marquée par la musique. Des cantigas particulières sont entonnées le matin, d'autres l'après-midi ou au crépuscule. D'autres encore sont interprétées avant les repas, au moment des ablutions, et ainsi de suite. Étant donné qu'il est interdit de parler pendant ce temps dans le roncó, ces chants représentent le moyen de communication le plus sûr pour les novices en réclusion.

L'ordre que la musique impose au déroulement temporel et à la succession des événements est bien illustré par la séquence des chants destinés aux divinités lors du rite public dans le barracão. Cette séquence des orixás invoqués (xirê) ordonne la cérémonie: les invocations musicales, les formules rythmiques qui les accompagnent au tambour, les salutations verbales, la danse et la représentation sont autant d'éléments qui, vus en rapport avec ce qui se passe directement dans le barracão, donnent, sous une forme de spectacle total, une image de la personnalité de tel ou tel orixá. Bien que la séquence des orixás invoqués lors du toque peut varier d'une congrégation à l'autre, il existe un certain consensus faisant que l'on commence toujours avec les chants adressés à Exu, suivis par ceux appelant Oxóssi, Ossaim ou Omolu, l'invocation à Oxalá terminant la série. Les invocations musicales s'adressant aux autres divinités - une quinzaine en tout - y sont insérées. Le principe organisateur de la musique dans le temps sacré du rite engage toute l'assemblée des fidèles, comme l'illustre la fréquence de remarques du genre: «Tu es juste à temps, le toque en est encore à Ogun». 


\section{Étendue du répertoire}

Pour le candomblé ketu, on ne dispose pas de données précises sur le volume global du répertoire fixe des chants et des formules rythmiques. A côté des chants pratiquées dans toutes les congrégations, qu'elles se situent à Bahia ou ailleurs, on rencontre aussi des cantigas peu répandues. Pour ce qui est du répertoire du tambour, il y a plus d'homogénéité, étant donné que les principaux toques sont connus de toutes les congrégations. Malgré cela il n'est pas toujours certain qu'un rythme représente une formule originale ou une variante. Dans la congrégation d'Ilê Omô Ogun (São Paulo), le chef religieux (pai-de-santo) a transcrit les paroles de quelque trois cents cantigas entonnées lors des cérémonies publiques organisées sous son égide. D'autre part, soixante-treize textes de cantigas provenant de rites se rapportant aux feuilles et aux herbes (sassanha) ont été notés. Il reste à transcrire des cantigas de roncó. Mais la maison d'Ilê Omô Ogun est un cas particulier, car les textes des cantigas se transmettent le plus souvent oralement dans le cadre de la congrégation.

\section{LA MUSIQUE DANS LA CONGRÉGATION D'YLE DE ERUME-FÁ: ÉTUDE DE CAS}

\section{Un rite public: le toque de bolar}

Le rôle de la musique apparait le plus clairement lors de rites publics tels qu'une cérémonie consacrée à un orixá ou un toque normal. Le rite décrit ci-après, qui se déroula en février 1991 dans la maison d'Ylê de Eruma-fá, était ce qu'on appelle un toque de bolar, destiné à l'accueil des novices voués à la réclusion. Je connais cette congrégation du Municipio de Santo Amaro (Bahia) et son chef, Pai Tidu, depuis une dizaine d'années en raison de mes recherches ethnologiques et musicologiques dans la région du Recôncavo de Bahia (cf. Oliveira Pinto 1991). C'est grâce à cette fréquentation que j'ai pu entreprendre une étude approfondie de ce candomblé . $^{\text {. }}$

Le toque d'Ylê de Erume-fá commença le samedi 3 février 1991 vers 22 heures et dura jusqu'au lendemain à trois heures du matin. Le tableau suivant contient le premier vers de chaque cantiga entonnée, ainsi que, en gras, l'appellation de la formule rythmique qui l'accompagnait. Dans la colonne du milieu, on trouve les divinités invoquées, et dans celle de droite, sous forme de mots-clefs, quelques observations à propos des pièces. Enfin, à l'extrême droite est indiqué de quel répertoire - ketu ou congo-angola - provient chaque pièce.

Une enquête musicologique à Bahia et à Pernambouc en 1991 fut effectuée grâce au financement de l'Institut international pour la musique traditionnelle (Berlin) et du Deutsche Musikrat (Bonn) dans le cadre d'un accord avec l'Universidade de São Paulo. 


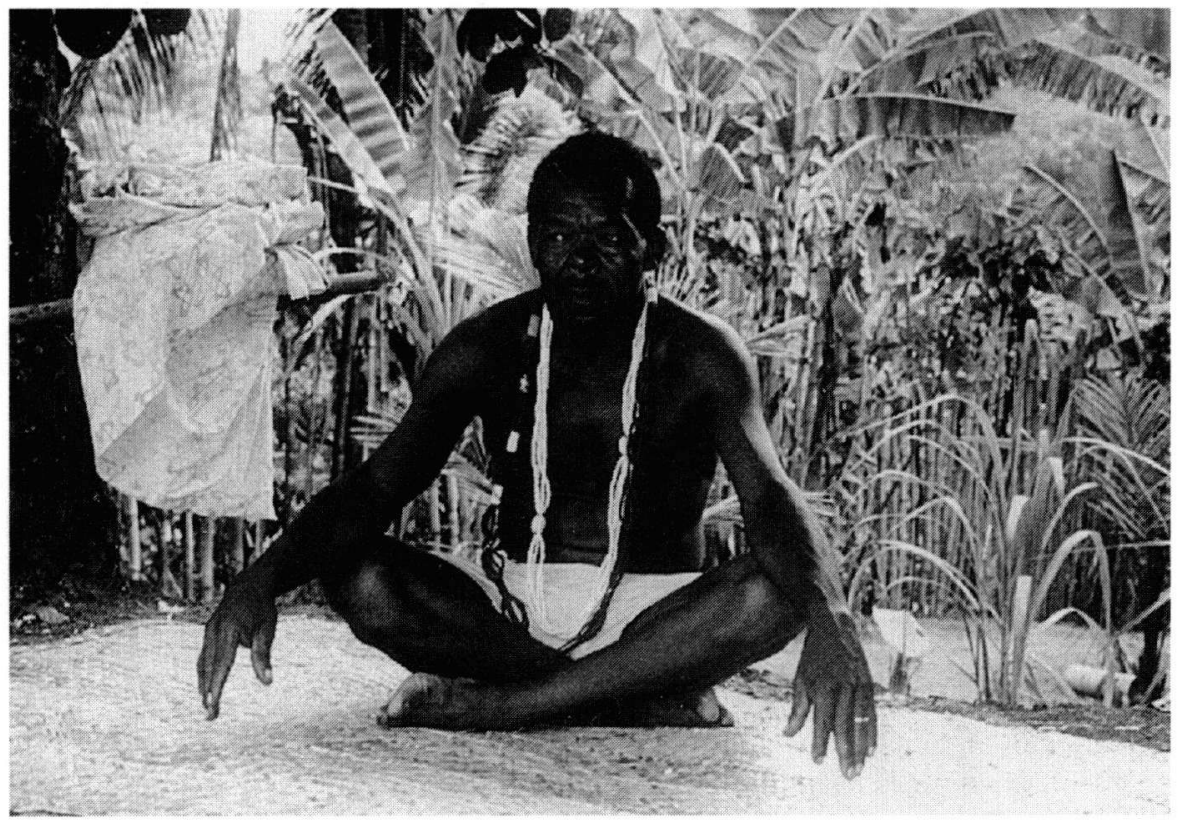

Fig. 1: Le chef religieux (pai-de-santo) Tidu, Pilar 1991.

Xirê et rum dans la Ylê de Erume-fá (Babalorixá Tidu) Pilar, Bahia, 3-4 février 1991

\begin{tabular}{|c|c|c|c|c|}
\hline $\mathbf{N}^{\circ}$ & Cantiga/toque & Pour & Remarques & Répertoire \\
\hline $\begin{array}{l}1 \\
2 \\
3 \\
4 \\
5\end{array}$ & $\begin{array}{l}\text { Ibarabô/vassá lento } \\
\text { Tiriri lonan/vassá } \\
\text { Ina ina/vassá } \\
\text { Um bará/ijexá } \\
\text { Exu onan/ijexá }\end{array}$ & $\begin{array}{c}\text { Exu } \\
" \\
" \\
" \\
"\end{array}$ & $\begin{array}{l}\text { normalement la } 2^{\mathrm{e}} \text { dans le xiré } \\
\text { cantiga rare }\end{array}$ & $\begin{array}{c}\text { Ketu } \\
\text { " } \\
\text { " } \\
\text { " }\end{array}$ \\
\hline $\begin{array}{l}6 \\
7 \\
8 \\
9\end{array}$ & $\begin{array}{l}\text { Onilé Aguê/vassá lento } \\
\text { Agué Marê/ } \underline{\text { vassá lento }} \\
\text { Tororo Aguê/vassá lento } \\
\text { Bom bom xé/ } \underline{\text { avaninha }}\end{array}$ & $\begin{array}{c}\text { Ossaim } \\
" \\
" \\
"\end{array}$ & $\begin{array}{l}\text { xirê de Ossaim } \\
" \\
" \\
\text { de «Ylê de Erume-fá» }\end{array}$ & $\begin{array}{l}" \\
" \\
"\end{array}$ \\
\hline $\begin{array}{l}10 \\
11 \\
12 \\
13 \\
14 \\
15 \\
16\end{array}$ & $\begin{array}{l}\text { Alujá } \\
\text { Tonibobé } \\
\text { Ilu } \\
\text { Obá nixá/vassá } \\
\text { Obá cossô/vassá } \\
\text { Aira aira/vassá } \\
\text { Aro lé/vassá }\end{array}$ & $\begin{array}{c}\text { Xangô } \\
" \\
" \\
" \\
" \\
"\end{array}$ & $\begin{array}{l}1^{\mathrm{er}} \text { cantiga } \\
3^{\mathrm{e}} \text { cantiga } \\
2^{\mathrm{e}} \text { cantiga } \\
5^{\mathrm{e}} \text { cantiga }\end{array}$ & $\begin{array}{l}\text { " } \\
\text { " } \\
\text { " } \\
\text { " } \\
\text { " }\end{array}$ \\
\hline
\end{tabular}




\begin{tabular}{|c|c|c|c|c|}
\hline $\begin{array}{l}17 \\
18 \\
19 \\
20\end{array}$ & $\begin{array}{l}\text { Aka keba/vassá } \\
\text { Omolu alaicó/vassá } \\
\text { Ejo ala ejo/vassá } \\
\text { Aua ejo/vassá }\end{array}$ & $\begin{array}{c}\text { Omolu } \\
" \\
"\end{array}$ & (du rum, pas du xirê) & $\begin{array}{l}\text { " } \\
\text { " } \\
\text { " }\end{array}$ \\
\hline $\begin{array}{l}21 \\
22 \\
23\end{array}$ & $\begin{array}{l}\text { Tinatina/ } \underline{\text { savalu-sató }} \\
\text { E xo xo/ } \underline{\text { bravum }} \\
\text { Ejo arauê/ } \underline{\text { bravum }}\end{array}$ & $\begin{array}{c}\text { Oxumaré } \\
" \\
"\end{array}$ & $\begin{array}{l}\text { cantiga caractéristique } \\
\text { cantiga rare } \\
\text { cantiga rare }\end{array}$ & $\begin{array}{l}" \\
"\end{array}$ \\
\hline $\begin{array}{l}24 \\
25 \\
26\end{array}$ & $\begin{array}{l}\text { Angorô/cabula } \\
\text { A iné/barravento } \\
\text { Angorô a saia/barravento }\end{array}$ & $\begin{array}{l}\text { Angorô } \\
\text { " }\end{array}$ & suit toujours la précédente & 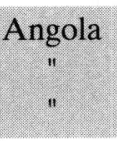 \\
\hline $\begin{array}{l}27 \\
28 \\
29\end{array}$ & $\begin{array}{l}\text { Roxo mocumbo/congo } \\
\text { Como senzala/congo } \\
\text { Beu beu/ } \underline{\text { congo }}\end{array}$ & $\begin{array}{l}\text { Ogun } \\
" \\
"\end{array}$ & $\begin{array}{l}1^{\mathrm{er}} \text { cantiga pour Ogun } \\
2^{\mathrm{e}} \text { cantiga du xirê }\end{array}$ & $\begin{array}{l}" \\
"\end{array}$ \\
\hline $\begin{array}{l}30 \\
31 \\
32 \\
33 \\
34 \\
35\end{array}$ & $\begin{array}{l}\text { Auenda canjira/cabula } \\
\text { Auê taua mim/cabula } \\
\text { Bambe ia/cabula } \\
\text { Adeus cutala/cabula } \\
\text { O seu Odé/ congo } \\
\text { Agué maré/ } \underline{\text { barravento }}\end{array}$ & $\begin{array}{l}\text { Oxóssi } \\
\text { " } \\
\text { " } \\
\text { " }\end{array}$ & $\begin{array}{l}\text { typique du xiré } \\
\text { suit toujours la précédente } \\
\text { cantiga du cand.-de-caboclo }\end{array}$ & $\begin{array}{l}\text { " } \\
\text { " } \\
\text { " } \\
\text { " }\end{array}$ \\
\hline $\begin{array}{l}36 \\
37 \\
38 \\
39\end{array}$ & $\begin{array}{l}\text { Quimemeso/cabula } \\
\text { Catende/cabula } \\
\text { Catende goma } / \text { b.vento } \\
\text { Cat. de ladigina } / \underline{\text { b.vento }}\end{array}$ & $\begin{array}{c}\text { Catendê } \\
\text { " } \\
" \\
"\end{array}$ & & $\begin{array}{l}" \\
" \\
"\end{array}$ \\
\hline $\begin{array}{l}40 \\
41 \\
42 \\
43 \\
44 \\
45\end{array}$ & $\begin{array}{l}\text { Cafunguê/congo } \\
\text { Cumbe cumbelaci/congo } \\
\text { E malê/congo } \\
\text { Sambuê/congo } \\
\text { Mona quera/ barravento } \\
\text { Alaketurê/vassá }\end{array}$ & $\begin{array}{c}\text { Tempo } \\
\text { " } \\
\text { " } \\
\text { " }\end{array}$ & $\begin{array}{l}\text { typique du xirê } \\
\text { cantiga rare } \\
\text { typique du xirêe } \\
\text { " } \\
\text { pas pour le xirê. Point fort (tournant): } \\
\text { estado de santo, bolar no santo }\end{array}$ & 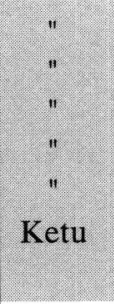 \\
\hline 46 & $\underline{\text { Avaninha }}$ & & $\begin{array}{l}\text { toque pour l'entrée et } \\
\text { la sortie du barracão }\end{array}$ & $"$ \\
\hline 47 & $(. .$.$) / cabula$ & & déplacement du bolados & Angola \\
\hline 48 & Agô lonã/bravum & 1 & $\begin{array}{l}\text { Entrée des orixás/santos dans } \\
\text { le barracão }\end{array}$ & Ketu \\
\hline 49 & Agô nilê/ vassá & 1 & Salutations des orixás & $"$ \\
\hline $\begin{array}{l}50 \\
51\end{array}$ & $\begin{array}{l}\text { Ogun bragadá/vassá } \\
\text { Ogun meje je/vassá }\end{array}$ & $\underset{1 "}{\text { Ogun }}$ & cantiga de guerra (de guerre) & $"$ \\
\hline
\end{tabular}




\begin{tabular}{|c|c|c|c|c|}
\hline $\begin{array}{l}53 \\
54 \\
55\end{array}$ & $\begin{array}{l}\text { Pa mi Ogun/vassá rápido } \\
\text { Mariwo laja/vassá } \\
\text { Aê logunjá/alujá } \\
\text { Axé xere/ ajagun/vassá }\end{array}$ & $\begin{array}{l}" \\
" \\
"\end{array}$ & $\begin{array}{l}\text { cantiga fort, seulement pour } \\
\text { l'orixá présent } \\
\text { cantiga de guerra dans alujá } \\
\text { cantiga typique }\end{array}$ & $\begin{array}{l}" \\
" \\
"\end{array}$ \\
\hline $\begin{array}{l}56 \\
57 \\
58 \\
59 \\
60 \\
61\end{array}$ & $\begin{array}{l}\text { Marabo ayô/bravum } \\
\text { Awode erecê/bravum } \\
\text { Ala ja were/bravum } \\
\text { Ero boawo/bravum } \\
\text { A koro/bravum } \\
\text { lemanjá ê/vassá }\end{array}$ & $\begin{array}{c}\text { Iemanjá } \\
\text { " } \\
\text { " } \\
\text { " }\end{array}$ & $\begin{array}{l}1^{\text {er }} \text { cantiga du xirê } \\
2^{\mathrm{e}} \text { cantiga du xirế } \\
\text { réponse au précédent } \\
\text { chanté quand l'orixá se manifeste } \\
\text { typique de Tidu }\end{array}$ & $\begin{array}{l}\text { " } \\
\text { " } \\
\text { " } \\
\text { " }\end{array}$ \\
\hline $\begin{array}{l}62 \\
63 \\
64 \\
65 \\
66 \\
67\end{array}$ & $\begin{array}{l}\text { Alujá } \\
\text { Tonibobe } \\
\underline{\text { Ilu }} \\
\text { Xangô ro to/vassá } \\
\text { Cô acolá/ alujá } \\
\text { Ko ko ko/ alujá }\end{array}$ & $\begin{array}{c}\text { Xangô } \\
" \\
" \\
" \\
" \\
"\end{array}$ & $\begin{array}{l}\text { cantiga fundamento de Tidu } \\
\text { cantiga fundamento de Tidu } \\
\text { typiquement «ketu» }\end{array}$ & $\begin{array}{l}" \\
" \\
" \\
" \\
"\end{array}$ \\
\hline $\begin{array}{l}68 \\
69 \\
70\end{array}$ & $\begin{array}{l}\text { E Angorô/cabula } \\
\text { Angorô sinho/cabula } \\
\text { Angorô a saia/barravento }\end{array}$ & $\begin{array}{c}\text { Angorô } \\
\text { " }\end{array}$ & cantiga du xirê & $\begin{array}{c}\text { Angola } \\
" ~\end{array}$ \\
\hline $\begin{array}{l}71 \\
72 \\
73 \\
74 \\
75 \\
76 \\
77\end{array}$ & 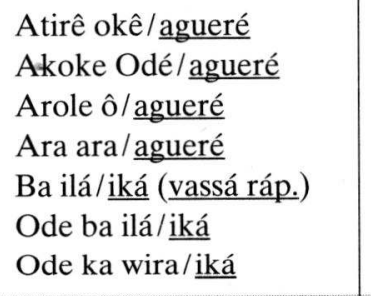 & $\begin{array}{c}\text { Oxóssi } \\
\text { " } \\
" \\
" \\
" \\
" \\
"\end{array}$ & $\begin{array}{l}1^{\text {er }} \text { dans le rum pour Oxóssi } \\
\text { précède le suivant } \\
\text { cantiga de la chasse } \\
\text { Tidu est saisi par son orixá (Iansã) }\end{array}$ & $\begin{array}{c}\text { Ketu } \\
" \\
" \\
" \\
" \\
" \\
"\end{array}$ \\
\hline $\begin{array}{l}78 \\
79 \\
80 \\
81 \\
82 \\
83 \\
84\end{array}$ & $\begin{array}{l}\text { Quebra prato } \\
\text { Oyá gueri/vassá lento } \\
\text { Awoyo do/vassá } \\
\text { Odo muyan/vassá } \\
\text { Kiba le le/ vassá } \\
\text { Bara eke/alujá } \\
\text { Elo Oyá/avaninha }\end{array}$ & $\begin{array}{c}\text { Iansã } \\
\text { " } \\
\text { " } \\
\text { " } \\
\text { " }\end{array}$ & $\begin{array}{l}\text { ouvre normalement le xirê } \\
2^{\mathrm{e}} \text { cantiga } \\
\text { en fait cantiga de funeral } \\
\text { rare, habituellement pour Xangô } \\
\text { seulement en présence de l'orixá }\end{array}$ & $\begin{array}{l}" \\
" \\
" \\
" \\
" \\
"\end{array}$ \\
\hline $\begin{array}{l}85 \\
86\end{array}$ & $\begin{array}{l}\text { Oyá kete/vassá rápido } \\
\text { Iyá ba orô/vassá }\end{array}$ & " & $\begin{array}{l}\text { cantiga pour Iyábalé } \\
\text { cantiga des iabás, où ne dansent } \\
\text { que des } \text { orixás féminins }\end{array}$ & " \\
\hline $\begin{array}{l}87 \\
88\end{array}$ & $\begin{array}{l}\text { Indeburê/cabula } \\
\text { Sinha vanju/congo }\end{array}$ & $\begin{array}{c}\text { Iansá } \\
\text { " }\end{array}$ & $\begin{array}{l}1^{\text {er }} \text { dans le rituel angola } \\
\text { peut aussi ouvrir le xirê }\end{array}$ & Angola \\
\hline 89 & Avaninha & 1 & adieu aux orixás & Ketu \\
\hline
\end{tabular}




\section{Xirê}

La première partie de la cérémonie religieuse ( ${ }^{\text {os }} 1$-45), appelée xirê, est introduite par des chants s'adressant à Exu, le médiateur entre le monde terrestre et l'univers des orixás. Au Brésil, Exu est particulièrement craint, car il n'est pas seulement un protecteur mais punit aussi celui qui n'a pas obéi aux prescriptions religieuses. C'est pourquoi, avant le début du rite proprement dit, on fait une offrande à Exu et on "prend congé de lui » (despachar), pour que la cérémonie puisse se dérouler sans incident ${ }^{3}$.

Depuis le début, le barracão est rempli de fidèles et de visiteurs. Comme la porte d'entrée reste ouverte sur l'extérieur, des visiteurs ne cessent d'arriver au cours de la cérémonie; ils prennent la place qui leur revient sur les bancs le long des deux murs latéraux de la salle. Au milieu de la pièce, les adeptes initiés forment un cercle; ils chantent et dansent en se déplaçant dans le sens contraire à celui des aiguilles d'une montre. Devant le mur du fond, où se trouve également le passage vers les pièces secrètes de la maison, sont entreposés les trois tambours sacrés, le grand rum, le rumpi moyen et le lé au son aigu. Les musiciens sont assis sur un banc surélevé derrière leurs instruments. A côté d'eux se tient le joueur de cloche double agogô. Pai Tidu et un ogã , un dignitaire de la congrégation, remplissent en alternance la fonction de meneur du chant.

Une fois les chants pour Exu terminés, Pai Tidu se lance dans le répertoire ketu destiné à Ossaim ( ${ }^{\text {os }}$ 6-9), la divinité des feuilles et des plantes médicinales, dont l'importance dans cette cérémonie tient au fait que les danseurs comptent parmi eux des novices qui devront, au cours de l'initiation, faire de nombreuses ablutions aux herbes infusées. Plus tard interviendront d'autres chants et formules rythmiques adressées à la même divinité, mais provenant du répertoire angola (où Ossaim s'appelle Catendê) (nºs 36-39).

D'autres orixás sont invoqués par la musique: Xangô (n $\left.{ }^{\text {ss }} 10-16\right)$, le maître du feu et de la foudre; Omolu ( $\left.{ }^{\text {os }} 17-20\right)$, l'«ancien ", l'orixá des maladies; Oxumaré (n ${ }^{\text {os }} 21-23$ ), l'hermaphrodite mythique dont les éléments sont l'eau et la terre. Puis le xirê passe au répertoire angola, toujours avec la même divinité qui s'appelle ici Angorô (n $\left.{ }^{\text {os }} 24-26\right)$. Suivent les cantigas à l'adresse d'Ogun (n $\left.{ }^{\text {os }} 27-29\right)$, le dieu guerrier du fer et des outils, ainsi que d'Oxóssi (n $\left.{ }^{\circ \mathrm{s}} 30-35\right)$, l'élégant chasseur maître de la forêt. Après l'invocation à la divinité des herbes (ici Catendê; $\mathrm{n}^{\text {os }} 36-39$ ) viennent les cantigas pour Tempo, une divinité vénérée seulement dans la liturgie congo-angola ( $\left.\mathrm{n}^{\mathrm{os}} 40-44\right)$. D’une manière générale, chaque cantiga entonnée pendant cette première partie de la cérémonie dure trois à quatre minutes.

Le jeu musical obéit à une stricte répartition sexuelle des rôles: les hommes jouent du tambour atabaque et, le plus souvent, de la cloche double agogô ou ga. En revanche, ce sont généralement les femmes haut placées dans la congrégation qui agitent la petite cloche à battant adjá. D'une manière générale, le

3 Pour l'«archétype» contradictoire d'Exu, voir par exemple Santos (1975), Trindade (1981) et Dopamu (1990) 


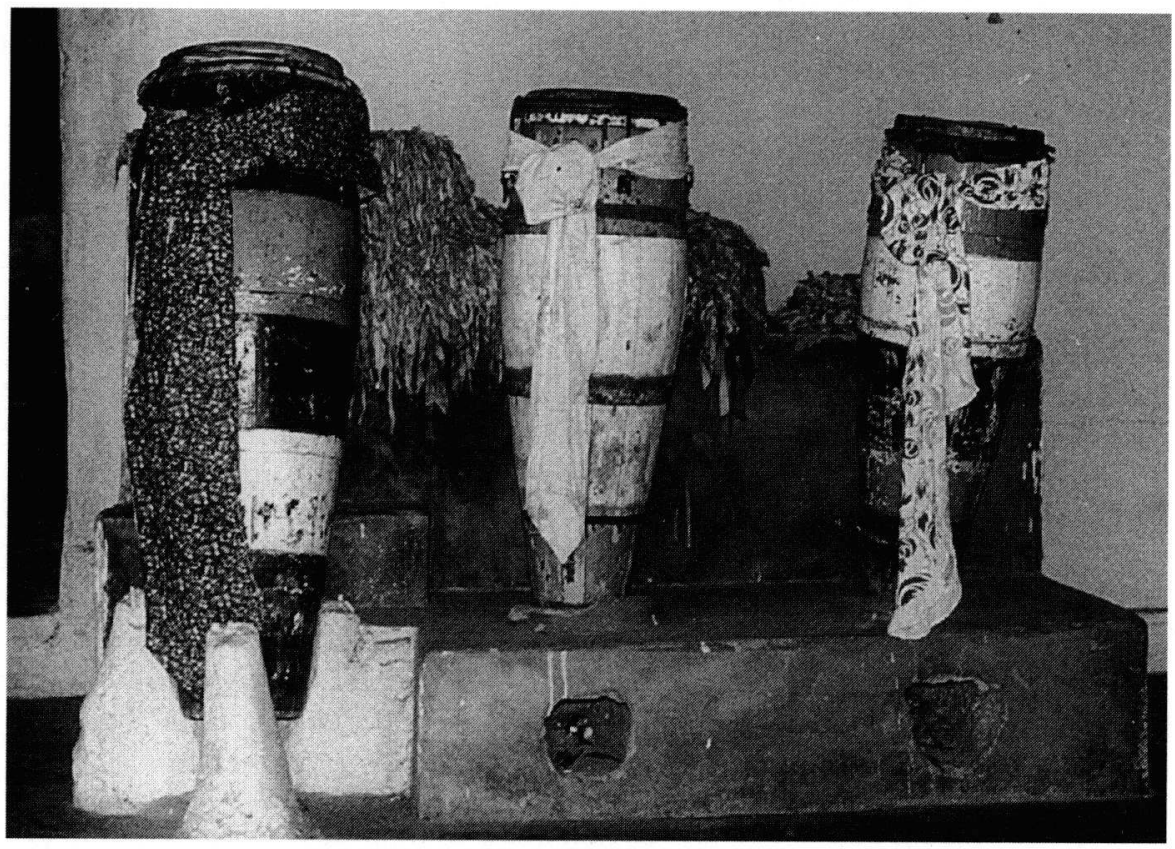

Fig. 2a: Les trois tambours sacrés rum, rumpi et lé.

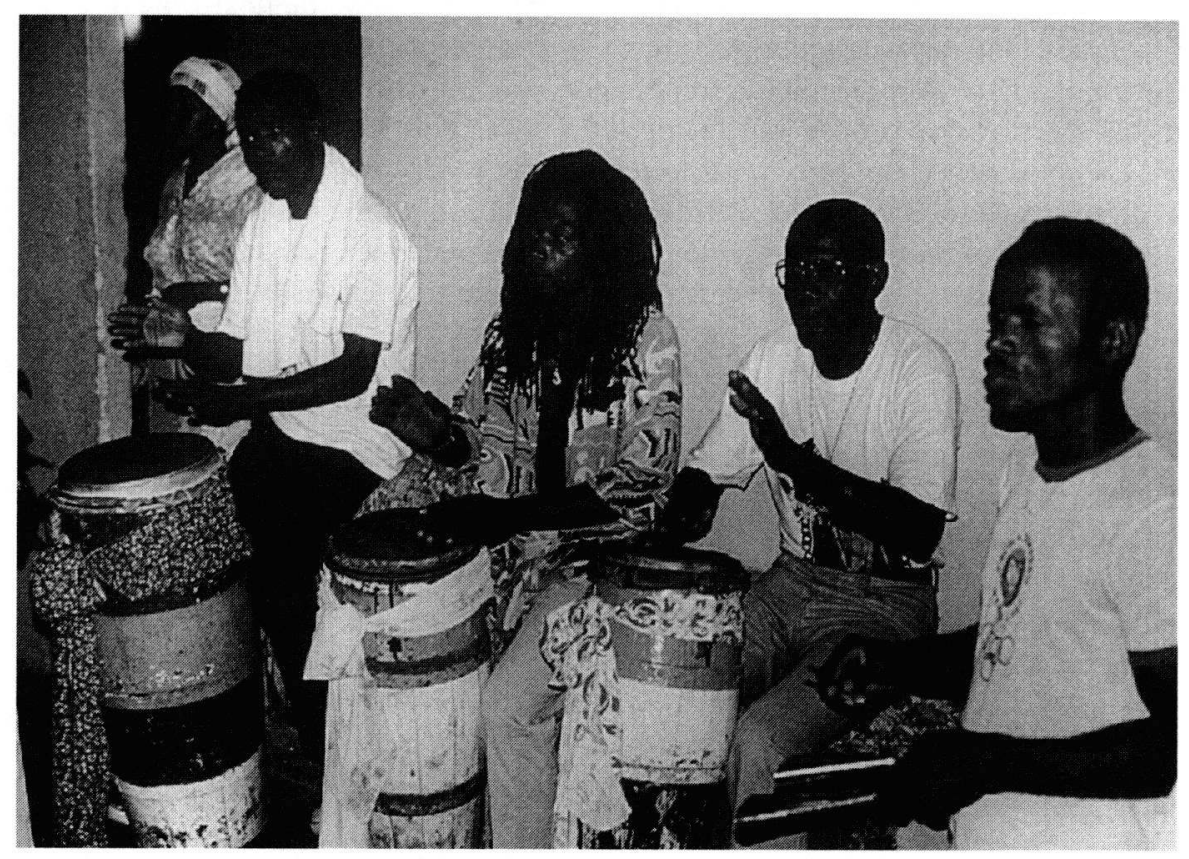

Fig. 2b: Tambourinaires (alabês) et joueur de la cloche double agogô lors du rite religieux dans l'Yle de Erume-fá. 
chant est exécuté par les hommes et les femmes; mais dans le chant alterné le chœur responsorial des femmes tend à prédominer.

\section{Les répertoires ketu et angola}

Un premier aperçu des cantigas et des toques passés en revue montre déjà un recours assez équilibré aux répertoires ketu et angola dans une seule et même cérémonie. Pour ce qui est du répertoire religieux, certains adeptes observent toutefois rigoureusement la division «nationale» des cultes du candomblé (nações), selon leur culture africaine d'origine: Bantous, Yoruba et Fon. Cela est faisable dans certaines congrégations de longue tradition, surtout à Salvador. Mais en de nombreux autres endroits, y compris la région du Recôncavo, on ne respecte guère une telle division, notamment lors des toques publics. Le déroulement du rite accompli dans l'Ylê de Erume-fá, tel que nous l'avons schématisé plus haut, en est un bon exemple. Nombreuses sont les divinités vénérées par la congrégation de Pai Tidu qui appartiennent au rite dit angola (bantou). Mais la liturgie ketu (yoruba) y joue un rôle tout aussi important, comme l'indique d'ailleurs le nom de la congrégation: Ylê équivaut au yoruba ile et signifie "maison». Les causes en sont d'ordre historique. Pai Tidu lui-même fut initié dans une congrégation d'obédience angola, mais il reprit plus tard la direction de l'Ylê de Erume-fá, jusque-là surtout d'obédience ketu. En tant que maître religieux, Pai Tidu assume la responsabilité de la liturgie ketu comme de la liturgie angola. Quelques mauvaises langues au sein de la congrégation prétendent cependant que sa manière de présenter le répertoire ketu est fortement influencée par le mode d'expression propre au rite angola.

La coexistence de deux répertoires religieux dans le candomblé, angola et $k e t u$, se manifeste dans le xirê, voire dans toute la cérémonie publique par la musique et les paroles des chansons, mais aussi par les danses en cercle et les représentations individuelles. Si la musique, les mouvements et les gestes expriment bien le caractère archétypal de chaque divinité, ils n'en font pas moins apparaître les divergences entre les répertoires ketu et angola. Une distinction claire selon le répertoire s'établit notamment entre les formules rythmiques jouées au tambour, sur le plan tant des structures musicales et rythmiques que de la technique de jeu. D'une manière générale dans le répertoire ketu, on frappe le tambour avec des bâtons (lé et rumpi avec deux bâtons, le grand tambour rum avec un bâton et une main nue). Dans les autres répertoires, on ne se sert que des mains nues. Dans le répertoire angola, trois toques de base - désignés par les termes de congo, barravento et cabula - déterminent l'évolution musicale, alors qu'il existe une vingtaine de toques différents dans le répertoire ketu. Ces formules rythmiques jouent un rôle encore plus important dans la seconde partie de la cérémonie ${ }^{4}$.

4 Pour ce qui est des formules mélodiques, de l'arrangement musical, de l'instrumentarium etc. dans l'un et l'autre répertoires, les critères les plus importants ont été résumés ailleurs (voir Oliveira Pinto 1991: 224, 225). 


\section{Présence de la sphère spirituelle sur terre: estado de santo et bolaçao}

Tout rite public du candomblé vise à établir un lien direct avec la sphère surnaturelle des orixás ou santos. La manifestation des divinités s'accomplit au travers des adeptes initiés de la congrégation. Au cours de la cérémonie, ceux-ci sont conditionnés pour qu'ils atteignent l'estado de santo, l'«état sacré ». Cet état de conscience particulier, que l'on a l'habitude de désigner, du point de vue de la culture occidentale, par «transe de possession», signifie, pour le fidèle, la capacité d'accueillir et de personnifier un orixá. Dans l'acte religieux, de nombreux facteurs concourent à cette fin. Répétons-le, un rôle de toute première importance revient à la musique: celle-ci suscite des associations que la congrégation comprend et auxquelles elle réagit de manière appropriée. Telle ou telle cantiga de fundamento (chant auquel on attribue un pouvoir particulier) ou encore telle ou telle cantiga de dar rum (entonnée le plus souvent en la présence des êtres spirituels) peut engendrer l'estado de santo, pour autant que certaines conditions rituelles soient réunies. Ainsi la cantiga interprétée au moment où un individu est «saisi» pour la première fois par un orixá (bolar no santo) conservera une signification spéciale pour lui toute sa vie: il y réagira toujours fortement et d'une manière personnalisée dans le contexte religieux.

En tant qu'état de conscience spécifique induit par l'initiation, l'estado de santo est une expérience individuelle dont la signification est étroitement liée à la conception religieuse de la «personnalité» dans le culte du candomblé (Goldmann 1987). En raison de son caractère individuel, il est malaisé de proposer une définition générale des mécanismes qui suscitent, puis entretiennent pendant quelque temps l'estado de santo ${ }^{5}$.

Un rôle important revient également aux paroles des cantigas dans l'induction d'une transe motivée religieusement. Même si, comme c'est notamment le cas du candomblé ketu, la terminologie religieuse et les chants sacrés, qui comprennent surtout des mots africains, résistent à une traduction littérale, on les comprend et l'on sait exactement ce qu'elles veulent dire et quel rôle leur revient d'après le moment de la cérémonie ${ }^{6}$. Gilbert Rouget a même affirmé que, dans les rites dits de possession, la signification des textes des chants excède en importance le rôle de la musique:

«La vertu de cette musique de possession réside non pas dans ses caractères musicaux mais dans ses paroles. Les paroles qui apostrophent le dieu, la personne, et dans une langue convenue généralement et très peu comprise des gens qui ne sont pas inités au culte. Ce sont ces paroles qui fonctionnent et non pas l'aspect apparemment exaltant de la musique, son aspect frénétique et très fortement rythmé qui est sensiblement le même que celui qui a cours dans des cérémonies qui n'ont rien à voir avec la transe et la possession» (Rouget 1984: 106).

5 On a tenté de le faire par référence à la musique. Voir par exemple Bastide (1945), Binon (1967), Béhague (1984) et Lühning (1990).

6 Pour une traduction et une analyse de quelques cantigas vouées à Exu, dont aussi notre pièce $\mathrm{n}^{\circ} 3$ Ina Ina, voir Santos (1975: 190). 
En effet, le caractère de fundamento d'une cantiga dépend en premier lieu de son contenu textuel et symbolique et non pas de traits exclusivement musicaux. Il n'en reste pas moins vrai qu'il existe dans le candomblé ketu un répertoire musical inconnu en dehors de la sphère religieuse, si bien que la fusion des textes sacrés avec cette musique particulière exerce une emprise particulière sur l'estado de santo et sur la première transe chez le non-initié.

Avec la séquence $\mathrm{n}^{\circ} 45$, Alaketurê, on arrive au cœur de la cérémonie : le mâ̂tre de l'Ylê de Erume-fá effectue une transition abrupte en entonnant cette cantiga de fundamento à Oxóssi, de provenance ketu. A considérer la suite des événements, on a ici un tournant, au sens de «liminas» (V. Turner). En entonnant cette cantiga, la plupart des danseurs atteignent l'estado de santo, alors que d'autres tombent subitement en transe (bolar no santo). La sphère spirituelle se manifeste au travers des danseurs: les initiés atteignent l'estado de santo et personnifient ainsi un orixá ou santo; les non-initiés sont comme saisis par une force invisible et jetés à terre. Les danseurs initiés qui agissent maintenant comme médiums (cavalo de santo) quittent la salle au rythme avaninha des tambours ( $\left.\mathrm{n}^{\circ} 46\right)$, afin de se préparer pour la seconde partie de la cérémonie et de revêtir l'accoutrement des orixás et leurs insignes.

Immédiatement après leur départ, on entend le rythme cabula, sur lequel les bolados sont portés à l'extérieur. Cette séquence $\left(n^{\circ} 47\right)$ dure plus de treize minutes: pendant ce temps, on soulève les personnes jetées à terre sous l'effet de leur première transe en observant certains gestes rituels et on les transporte à l'extérieur du barracão, devant la porte du roncó. Les bolados sont enveloppés de tissus blancs: maintenant que leur santo s'est manifesté, ils sont appelés à subir l'initiation dans l'Ylê de Eruma-fá, qui durera plusieurs semaines. C'est surtout pour eux que le toque de bolar du 3 février 1991 avait été organisé.

Les trois séquences centrales de tout le rite, soit nos 45,46 et 47 , sont une nouvelle illustration éloquente de la présence simultanée d'un double héritage ketu et angola dans l'Ylê de Eruma-fá: lors de l'Alakaturê, une cantiga de fundamento à l'adresse d'Oxóssi, la sphère spirituelle se manifeste doublement (estado do santo et bolar no santo). Ensuite, les danseurs en estado de santo quittent la pièce sur un accompagnement provenant du répertoire ketu (rythme avaninha), tandis que le transfert des bolados se fait sur l'un des rythmes les plus importants du répertoire angola, à savoir le cabula.

\section{Lieux du rite}

Les deux principaux lieux rituels d'un toque, soit la salle principale (barracão) et la pièce secrète (roncó) sont localisés précisément dans l'esprit des participants et des visiteurs: en quittant le barracão, on parle d'entrada (entrée) dans le roncó; lorsque les santos reviennent plus tard dans le barracão, on parle de saída (sortie). Ce n'est donc pas le lieu le plus visible - la salle principale - qui est con- 


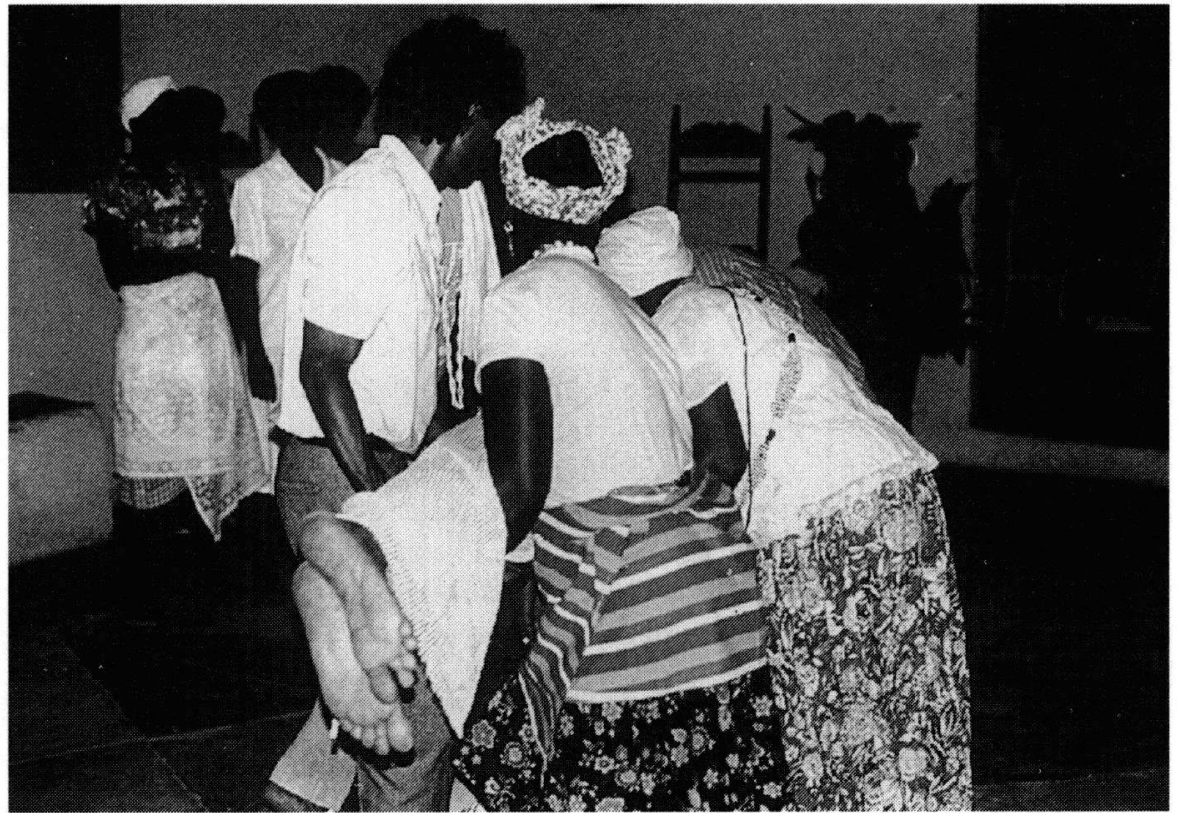

Fig. 3: Un bolado est porté hors de la salle principale par les assistants.

sidéré comme le centre d'après lequel on s'oriente et dans lequel on se trouve soi-même; le centre et le point de départ "géographique» au cours du toque, c'est le huis clos, le roncó. Voici la manière dont la plupart des participants s'imaginent le centre «géographique» de la cérémonie:

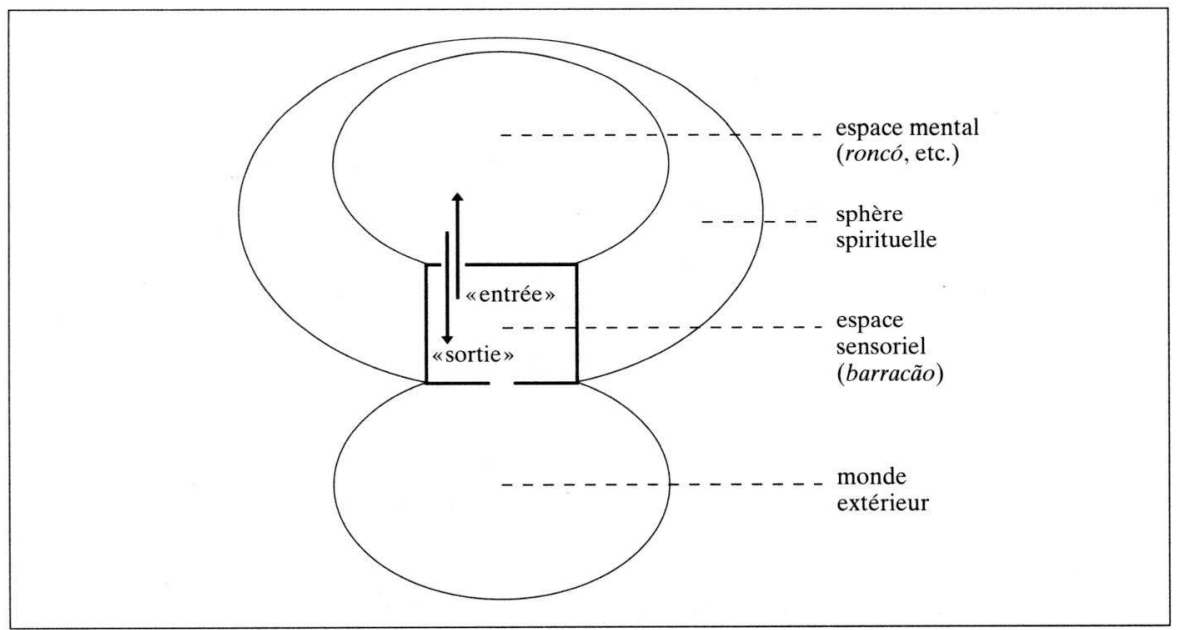

Fig. 4: Représentation schématique du lieu du rituel dans la cérémonie publique du candomblé. 
Dans le barracão, qui est le domaine de la perception sensorielle, la musique se manifeste dans ses dimensions acoustiques et impulsives, tandis que seul son axé (son énergie, sa force spirituelle) pénètre dans la sphère surnaturelle.

\section{Dar rum: la danse des divinités}

L'objectif de ce rite spécifique, qui est d'induire une première transe encore inarticulée chez certaines personnes, fut atteint après la pièce Alaketurê. La sphère spirituelle s'était toutefois manifestée, et c'est elle qui sera au centre des événements qui vont suivre, car une cérémonie publique vise en dernière analyse à attirer la présence des divinités dans la salle principale.

La seconde partie du toque commence par la sortie des santos qui, vêtus et ornés selon la circonstance, jaillissent maintenant dans la salle principale. C'est durant cette phase qu'une relation particulièrement étroite se noue entre les santos qui dansent et les tambourinaires, surtout le joueur du grand rum. Ce n'est pas un hasard si l'on parle ici de tomar rum ou dar rum, c'est-à-dire de «donner » la musique du rum et du reste de l'ensemble aux santos présents à travers les médiums initiés.

Les orixás suivants apparurent dans la seconde partie de la cérémonie de l'Ylê de Erume-fá:

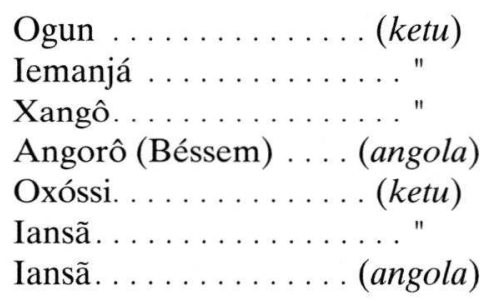

Ces santos dansaient, et on entendait les cantigas et les toques qui leur étaient propres. A la différence du xirê, le dar rum se caractérise par la danse individuelle, à l'exception de la séquence ${ }^{\circ} 86$, qui s'adresse à toutes les orixás féminines, ainsi que du rythme avaninha de la fin $\left(\mathrm{n}^{\circ} 89\right)$ pendant lequel tous les danseurs présents, y compris ceux qui ne sont pas en estado de santo, dansent en cercle dans le sens contraire à celui des aiguilles d'une montre avant de quitter la salle principale.

\section{Le grand tambour rum}

L'importance primordiale du grand tambour cylindrique appelé rum tient à sa fonction musicale et à son rôle dans la danse. Tandis que les deux tambours plus petits (lé et rumpi) servent à exécuter un ensemble de formules fixes souvent 


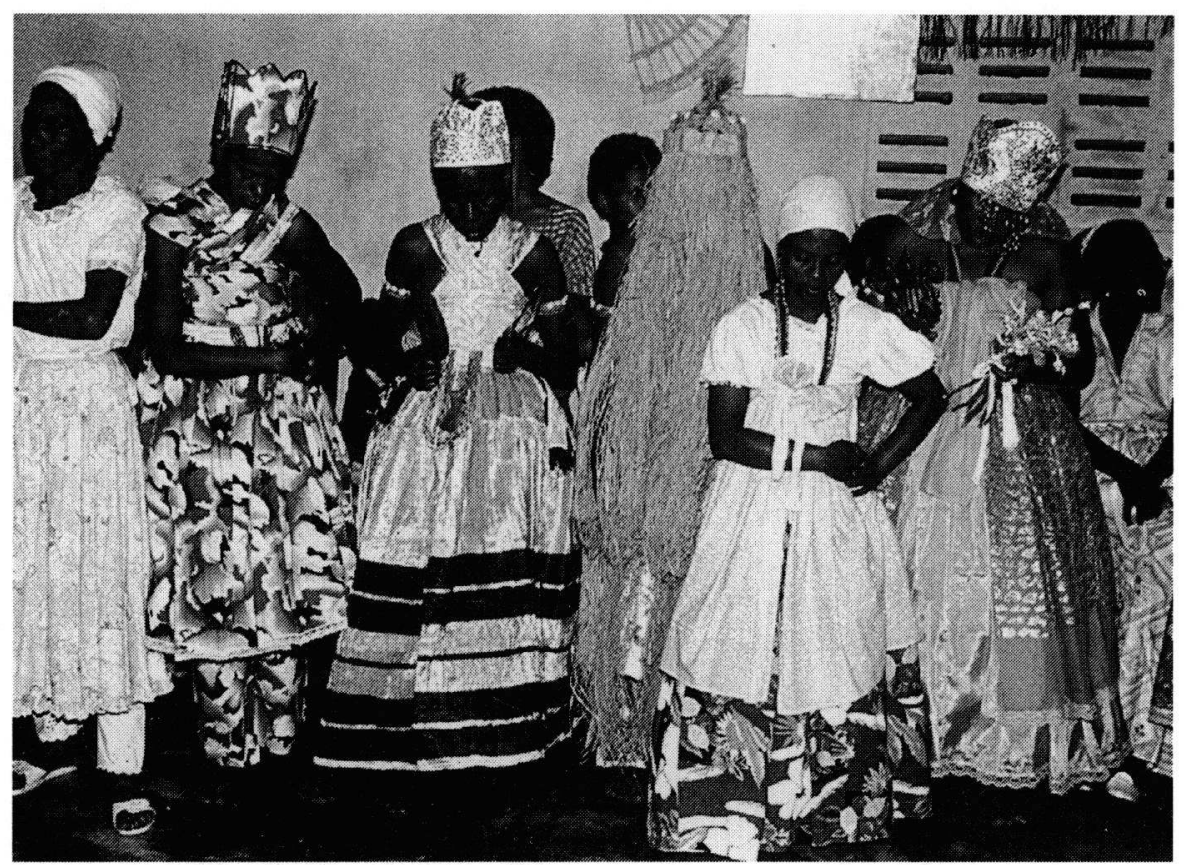

Fig. 5: Présence des orixás dans la salle principale.

identiques, donnant peu de place à l'ornementation, le rum ne marque dans un premier temps que la structure du toque, pour le faire ensuite varier musicalement et élaborer des phrases rythmiques complexes au timbre mélodique. Cela se passe en rapport étroit avec la danse des santos. La stricte division des tâches musicales au sein du toque dépend des hauteurs de son différentes des tambours: le rôle de marquer les temps revient aux instruments au son aigu; en revanche, les frappes amorties exécutées avec la main nue sur le rum pour marquer les temps faibles des tambours aigus, entretiennent un rapport de tension permanent avec les autres instruments, et parfois aussi avec le chant. Du point de vue de la technique de jeu, le rum est l'instrument le plus complexe des trois tambours.

Certaines frappes et certains effets sonores permettent à l'alabê, le meneur jouant du rum, d' «appeler» les santos qui dansent. Mais ici ce n'est pas le musicien qui occupe le devant de la scène; c'est l'instrument qui est personnifié car, pour les danseurs, c'est le rum qui les «appelle» et les contraint à danser jusqu'à l'épuisement. Le rum parvient également à «retenir» les santos dans la salle principale. Mais pour leur part les orixás, lorsqu'ils «sont sur terre» (estar em terra), c'est-à-dire qu'ils dansent au cours de la cérémonie publique dans le barracão, peuvent contraindre les alabês à exécuter certaines formules rythmiques. Cet échange est si intense qu'il arrive même qu'un santo s'arrête de danser parce qu'il n'apprécie pas le jeu des musiciens. 


\section{Les formules rythmiques (toques)}

Le répertoire ketu comprend des toques spécifiques, diversement structurés, qui correspondent au caractère de chaque orixá. Certains d'entre eux sont si étroitement liés à la personnalité de l'orixá qu'il n'est pas besoin de chant: pour Xangô, c'est alujá-tonibobé, pour Omolu, opanijé, pour Iansã, daró ou quebra prato. Il est plutôt rare d'entrendre de tels toques lors du xirê (cf. $\left.\mathrm{n}^{\text {os }} 10-12\right)$, car les santos ne sont pas encore présents pendant celui-ci. Dans le cas des pièces mentionnées, m'ont expliqué par la suite les musiciens, on a voulu souligner qu'à ce stade du xirê, c'est le répertoire de Xangô qui aurait dû être exécuté.

La cérémonie de l'Ylê de Erume-fá présente un certain nombre de toques que l'on peut regrouper selon des critères formels, soit la durée d'un cycle de 6 , 8,12 ou 16 unités de temps minimales. Ceux-ci sont caractéristiques de tout le répertoire ketu. Les formules rythmiques décrites ci-dessous correspondent chacune à la partie de la cloche double agogô, qui les suit pendant toute la durée de l'événement musical. C'est d'après elles que s'orientent les autres instrumentistes et les chanteurs.

$$
\begin{aligned}
& x=\text { frappe } \\
& .=\text { pause }
\end{aligned}
$$

(6)

$\begin{array}{ll}\text { Bravum } & x \cdot x \times \\ \text { Savalu/sató } & x . x \times x .\end{array}$

(8)

$\begin{array}{ll}\text { Agueré } & x x \ldots x x x . \\ \text { Agueré de Oxóssi } & x x \ldots x x x . \\ \text { Tonibobé } & x . x . x . x . \\ \text { [Adarrum] } & x . x . x . x .\end{array}$

Toque de Ketu/vassá $\mathrm{x} . \mathrm{x} x . \mathrm{X} . \mathrm{x} . \mathrm{x} \mathrm{x}$.

Toque de Ogun $\quad X . X . X X . X . X . X$

Alujá $\quad x \cdot x \cdot x \cdot x \cdot x \cdot x$.

[Ibi]

[Gincá]

(16)
Avaninha/vamunha
$x \ldots x \ldots x \ldots x . x \ldots$
Quebra prato/daró
$x \cdot x \cdot x \cdot x \cdot x \cdot x \cdot x \cdot x$
Ijexá
$x \cdot x \cdot x, x \cdot x \cdot x \cdot x .$.
[Opanijé]
$x x x \cdot x x x \cdot x x \cdot x \cdot x x$ 
Les toques en italique sont ceux qui accompagnent le plus souvent les cantigas. Entre crochets figurent d'autres rythmes importants qui furent cependant absents du rite étudié.

\section{Conclusion}

La fonction de la musique et les associations qu'elle crée doivent être évaluées diversement selon les étapes du toque de bolar de l'Ylê de Erume-fá, décrit cidessus. Dans la première partie, le xirê (jusqu'au $n^{\circ} 45$ ), la musique sert avant tout à invoquer et à exalter (louvação) les différents orixás ou santos. Les cantigas servent ici de supports musicaux à des contenus textuels, et elles structurent le déroulement du rite. En organisant une certaine étape de l'activité sacrée, le rite reste prévisible et correspond dans une large mesure aux attentes de la congrégation présente. Avec les mouvements de danse et le contenu des textes, la musique symbolise dans le xirê les mythes (lendas) relatifs aux orixás. Dans leur contexte rituel correspondant, certaines cantigas (mélodie et texte) amènent les initiés à l'estado de santo. Au moment de l'Alaketurê ( $\left.{ }^{\circ} 45\right)$, qui représente dans l'Ylê de Erume-fá une des principales cantigas de fundamento, cela survient d'une manière particulièrement abrupte. Le tournant du rite est, pour ainsi dire, forcé par la musique.

Dans la seconde partie du toque, le dar rum, la sphère spirituelle est présente dans la salle principale (santo em terra). La musique et la danse sont maintenant des éléments actifs de la présence des orixás. Ceux-ci ont pour moyen d'expression, non seulement les mouvements de danse, mais encore la musique, car l'activité des musiciens est souvent dirigée par la volonté des orixás présents.

A ce stade, la musique ne peut plus être abstraite du rite. L'ensemble de celuici se mue dans ses diverses dimensions en un contexte formalisé, où l'énergie de la musique se déploie dans les limites prescrites par la norme. Baragunã de Exu, l'un des musiciens de candomblé les plus réputés de São Paulo, l'exprime ainsi:

«La musique du dar rum est en elle-même si puissante que les prescriptions rituelles n'existent que pour canaliser l'énergie qu'elle renferme».

Comprise dans son sens le plus général, la musique structure donc le temps sacré; elle éveille des associations concrètes, elle décrit la mythologie des orixás; elle exalte et "appelle» la sphère spirituelle, et elle stimule dans son contexte rituel l'estado de santo. En présence des orixás et des santos, elle échappe toutefois aux aspects formalisés pour devenir elle-même un mythe vivant et une énergie active: dès lors les normes régissant le rite ne représentent plus qu'une limitation de l'espace et du temps. 


\section{Bibliographie}

\section{BASTIDE Roger}

1945 Imagens do Nordeste Místico em Branco e Preto. Rio de Janeiro: Cruzeiro.

1961 O Candomblé da Bahia. São Paulo. Ed. Nacional.

\section{BÉHAGUE Gerard}

1984 «Patterns of Candomblé music performance: an Afro-Brazilian religious setting», in: Performance Practice. Ethnomusicological Perspectives (G. Béhague Ed.). Westport/London: Greenwood Press, p. 231-54.

\section{BINON Gisèle}

1967 «La musique dans le candomblé», in: La musique dans la vie (T. Nikiprowetzky éd.). Paris: Office de coopération radiophonique, p. 159-207.

COSTA LIMA Vivaldo da

1976 «O conceito de nação nos candomblés da Bahia ». Afro-Asia 12: 65-90.

DANTAS Beatriz Góis

1988 Vovó Nagô e Papai Branco. Üsos e abusos da Africa no Brasil. Rio de Janeiro: Graal.

DOPAMU P. Ade

1990 Exu. O Inimigo invisivel do Homem. São Paulo: Editora Oduduwa.

\section{GOLDMANN Márcio}

1987 «A construção ritual da pessoa: a possessão no candomblé», in: Candomblé. Desvendando Identidades (C.E. Marcondes de Moura Ed.). São Paulo: EMW Editores, p. 87-119.

LÜHNING Angela

1990 Die Musik im candomblé nagô-ketu. Hamburg: K.D. Wagner.

PINTO Tiago de Oliveira

1991 Capoeira, Samba, Candomblé. Afro-brasilianische Musik im Recôncavo, Bahia. Berlin: Dietrich Reimer Verlag [Museum für Völkerkunde Berlin; N.F. 52]

ROUGET Gilbert

1984 «La musique et la transe: entretien avec Gilbert Rouget» Recherche, Pédagogie et Culture 65/66: 99-107.

SANTOS Juana Elbein dos

1975 Os Nagó e a Morte. Petrópolis: Vozes.

TRINDADE Liana Salvia

1981 «Exu: poder e magia», in: Olóorisà. Escritos sobre a Religião dos Orixás (C.E, Marcondes de Moura Ed.). São Paulo: Agora, p. 1-10.

\section{VERGER Pierre}

1955 «Première cérémonie d'initiation au culte des orishas nagô à Bahia au Brésil». Revista do Museu Paulista n.s. 9: 269-91.

1981 Orixás. São Paulo: Corrupio. 